\section{Similar effects of two modified constraint-induced therapy protocols on motor impairment, motor function and quality of life in patients with chronic stroke}

\author{
Wilma Costa Souza, 1 \\ Adriana B. Conforto, ${ }^{2}$ \\ Marco Orsini, 3 Annette Stern, 4 \\ Charles André 5 \\ 1Federal University of Rio de Janeiro, \\ Rehabilitation Center of Rio de Janeiro, \\ Brazil; 2Neurostimulation Laboratory, \\ Hospital das Clínicas/São Paulo \\ University, Brazil; ${ }^{3}$ Neurology \\ Department, Centro Universitário \\ Augusto Mottta, UNISUAM, Bonsucesso, \\ Brazil; 4School of Psychology, University \\ of Surrey, Guildford, UK; ${ }^{5}$ Department of \\ Neurology, School of Medicine, Federal \\ University of Rio de Janeiro, Brazil
}

\section{Abstract}

Modified constraint-induced movement therapy (CIMT) protocols show motor function and real-world arm use improvement. Meanwhile it usually requires constant supervision by physiotherapists and is therefore more expensive than customary care. This study compared the preliminary efficacy of two modified CIMT protocols. A two-group randomized controlled trial with pre and post treatment measures and six months follow-up was conducted. Nineteen patients with chronic stroke received 10 treatment sessions distributed three to four times a week over 22 days. CIMT3h_direct group received 3 hours of CIMT supervised by a therapist $(\mathrm{n}=10)$ while CIMT1.5h_direct group had 1.5 hours of supervised CIMT+1.5 hours home exercises supervised by a caregiver $(n=9)$. Outcome measures were the Fugl-Meyer Assessment, the Motor Activity Log, and the Stroke Specific Quality of Life Scale. The modified CIMT protocols were feasible and well tolerated. Improvements in motor function, real-world arm use and quality of life did not differ significantly between treated groups receiving either 3 or 1.5 hours mCIMT supervised by a therapist.

\section{Introduction}

Global ageing is associated with an everincreasing number of elderly individuals at increased risk for stroke.1,2 Stroke is a condi- tion with high incidence and mortality, and the most common cause of disability worldwide. 3 Most strokes occur in developing countries, 4,5 presenting a major burden to health care.

In stroke patients, recovery of the upper limb is often limited when compared with that of the lower limb. ${ }^{6}$ Loss of upper limb function significantly contributes to overall disability.7,8 Constraint-induced movement therapy (CIMT) is an evidence-based therapy for upper limb rehabilitation that evolved from basic research.9,10 The original protocol involved training of the upper limb by shaping and task practice for 6 hours per day and constraint of the unaffected hand for approximately $90 \%$ of waking hours during two weeks. ${ }^{10}$ The effectiveness of CIMT in post-stroke patients has been shown by randomized clinical trials.11-13 CIMT improves real-world arm function significantly more than customary care, or no treatment, in the chronic phase after stroke.

A limitation to widespread use of CIMT in stroke rehabilitation is that the original CIMT protocol requires constant supervision by physiotherapists during several hours per day, and is therefore more expensive than customary care. ${ }^{14}$ CIMT protocol is very difficult to implement under conditions of developing countries where most strokes occur. Modified CIMT protocols that include less time of direct supervision by a therapist would be more appropriate in developing countries that rely mostly on outpatient rehabilitation.

In recent years, several studies adapted the original protocol, $, 6,15-19$ and showed that modified protocols can also enhance motor function and real-world arm use. ${ }^{19}$ However results of modified protocols are conflicting. Supervised CIMT from 30 minutes to 3 hours over 10 sessions, ${ }^{14-18}$ led to enhancement of upper limb function in some, 15,18 but not all studies. ${ }^{14,17}$

The present study compares, in a public health facility in Brazil, the preliminary efficacy of two modified CIMT protocols, applied over ten sessions, with direct supervision provided by a therapist for either 3 or 1.5 hours. CIMT durations were based on feasibility of supervised treatment in middle- and low-income countries. The 3-hour supervised protocol was shown to be beneficial to upper limb function in previous studies, 14,17 while the 1.5 hour approach had not been previously tested. We hypothesized that both programs would lead to comparable changes in motor outcomes and quality of life.

\section{Materials and Methods}

\section{Study design}

In this pilot, randomized, single-blinded clinical trial we compared the feasibility, safety, and efficacy of 10 sessions of either 3 hours of CI-
Correspondence:Marco Orsini, Programa de PósGraduação em Ciências da Reabilitação, Praça das Nações, 34, Bonsucesso, Rio de Janeiro, CEP: 21041021, Brasil.

E-mail: orsinimarco@hotmail.com

Key words: modified constraint-induced therapy, motor impairment and function, quality of life, stroke.

Contributions: the authors contributed equally.

Conflict of interest: the authors declare no potential conflict of interest.

Received for publication: 9 April 2014. Accepted for publication: 3 January 2015.

This work is licensed under a Creative Commons Attribution NonCommercial 3.0 License (CC BYNC 3.0).

CC Copyright W. Costa Souza et al., 2015

Licensee PAGEPress, Italy

Neurology International 2015; 7:5430

doi:10.4081/ni.2015.5430

therapy supervised by a therapist $\left(\right.$ CIMT $_{3 \mathrm{~h} \text { direct) }}$, or 1.5 hours of supervised CItherapy +1.5 hours of home exercises supervised by a caregiver ( CIMT $_{1.5 \mathrm{~h} \text { direct }}$ ), in patients $>24$ months after stroke. In both groups, patients were instructed to restrain the affected limb during waking hours, for 10 days.

\section{Participants}

Inclusion and exclusion criteria were based on previous CIMT research. ${ }^{12}$

Inclusion criteria: age $>18$ years; history of ischemic or hemorrhagic stroke leading to upper limb paresis in the previous 24 months; minimal active range of motion of 10 degrees for wrist extension, 10 degrees for abduction/extension of the thumb and at least 2 additional digits, 90 degrees for shoulder flexion and abduction, 45 degrees for shoulder external rotation, 30 degrees for elbow extension, 45 degrees for forearm supination and pronation (from neutral position), wrist extension (from neutral), and finger extension of all digits; amount-of-use score on the Motor Activity $\log >2.5 ;{ }^{9}$ balance and stability to move using a glove in the unaffected hand; safe and independent transfer to toilet; ability to stand for two minutes with and without the glove (with support of upper limbs, if necessary); availability of a family member to supervise home exercises.

Exclusion criteria: medical problems or cognitive deficit (mini mental status examination score $<24)^{20}$ that could interfere with study completion; aphasia or hemineglect; intended or actual participation in any other study; significant pain ( $\geq 4$ in the visual analog scale) in 
any joint; upper limb treatment with anti-spasticity drugs in the previous 6 months; and severe upper limb spasticity ( $\geq 3$ in the Modified Ashworth Scale). ${ }^{21}$

The protocol was approved by the institutional review board for human studies of Federal University of Rio de Janeiro. Stroke patients were recruited in a public outpatient rehabilitation center and gave informed written consent to participate.

\section{Baseline measures}

The following characteristics were evaluated at baseline: gender, age, time from stroke, type of stroke, handedness and side of hemiparesis.

\section{Experimental design}

Patients were randomized to one of the two groups by a staff member not involved in the study. Randomization information was stored in sealed envelopes that were kept in a cabinet accessible solely to the principal investigator. Each patient received 10 treatment sessions distributed three to four times a week over 22 days. In the $\mathrm{CIMT}_{1.5 \mathrm{~h} \_ \text {direct }}$ group, patients performed exercises with the paretic upper limb for 1.5 hours at an outpatient facility and home exercises, supervised by a caregiver or family member, for additional 1.5 hours. Two days before treatment started, the caregiver was trained for one hour by the researcher providing CIMT on how to supervise the prescribed exercises performed by the patient at home. Each caregiver was instructed to make notes in a log book about the exercises performed, the number of repetitions and difficulties experience by the patient. At the beginning of each session the homework was discussed and when necessary, the level of difficulty was increased or new tasks were prescribed.

Patients in the $\mathrm{CIMT}_{3 \mathrm{~h} \text { _direct }}$ group performed exercises under direct supervision of the same therapist that treated patients in the CIMT $_{1.5 \mathrm{~h} \text { direct }}$ group for 3 hours per session, at the outpatient facility.

In both groups, training was provided in an individual basis and consisted of shaping principles, as outlined by Taub et al., 10,22 and task specific practice. Shaping exercises comprised a battery of tasks including grasping and releasing objects of different shapes, playing cards and board games, clay activities, drawing and painting. Tasks were tailored to needs of each patient. Task-specific practice for both groups involved preparing a snack (sandwiches and juice), including arranging dishes and cutlery on a table, washing and drying them, and putting them in a cupboard. Treatment regimens were designed to ensure that both groups received the same amount of task practice and shaping.

In both groups, patients were required to use a padded mitt in the unaffected hand at home, as much as possible during waking hours. The mitt prevented use of the unaffected hand to perform fine motor activities and should be used during activities of daily living (ADL) and household activities. The CIMT $_{1.5 \mathrm{~h} \text { _direct }}$ group received written assignment of practice at home. All patients were instructed to record the use time of the mitt and any difficulties perceived at home, in log books. At the beginning of each outpatient session the notes were discussed and, if necessary, problem-solving strategies were applied.

\section{Outcome measures \\ Primary outcome}

The primary outcome measure was the Motor Activity Log (MAL). ${ }^{9}$ The MAL was developed to measure the use of the affected arm in real world, ${ }^{23,24}$ and consists of a semi-structured interview that can be applied to both patients and caregivers. It is capable of detecting phenomena not evaluated in other instruments, such as the learned nonuse. ${ }^{24}$ The present study applied the original version (14 activities) of the MAL quality of movement scale (QOM). Although the original instrument presents two subscales, i.e., quality of movement (QOM) and amount of use (AOU), it has been shown that the QOM scale is able to capture components of both quality and quantity of use of the affected extremity in stroke patients. ${ }^{25}$ The MAL-14 presents a 5point ordinal scale and individuals are asked to rate how well they have used the affected arm in the past week. Higher scores reflect great quality of movements of the more affected arm in real world. 25

\section{Secondary outcomes}

Secondary outcomes included the FuglMeyer Assessment of Sensorimotor Recovery after Stroke (FMA) ${ }^{26-28}$ and the Stroke Specific Quality of life Scale (SSQOL). ${ }^{29-31}$

The FMA measures the level of motor impairment in stroke patients (best possible score for the upper limb, 66) and has excellent test-retest reliability and construct validity.

The SSQOL assesses health-related quality of life in stroke patients and encompasses three dimensions of the International Classification of Functioning, Disability and Health: impairment, activity and participation. 31,32 The instrument has proven its validity, reliability and sensitivity. It includes 49 items dealing with energy, family role, language, mobility, mood, personality, self-care, social role, reasoning, upper limb function, vision, and work/productivity. Each item is rated in a 5 -point scale and the scale has a maximal score of 245 points with higher scores meaning better health-related quality of life. Outcomes were assessed at baseline, two days after the intervention program (end of treatment) and 6 months after end of treat- ment by a trained physiotherapist blinded to group assignment.

\section{Analyses}

In order to compare treatment effects between the two groups, the Effectiveness Index - EI for MAL, ${ }^{33}$ FMA and SSQL was calculated in each group, comparing results before and post-treatment and before and 6 months after treatment using the following formula:

\section{$\mathrm{EI}=\frac{(\text { Total test score at T1 })-(\text { Total test score at T2 })}{\times 100}$ \\ (Maximum test score) - (Total test score at T1)}

The Effectiveness Index (EI) takes into account the fact that potential improvement for patients with high initial scores is lower than that for those with low initial scores. Thus, the EI reflects the proportion of potential improvement that was actually achieved during treatment. 34,35

After calculating the IE for each of the variables, Mann-Whitney U tests were applied to detect significant differences in treatment effects between the two groups, immediately after treatment and six months later $(\mathrm{P} \leq 0.025$, Bonferroni correction). Statistical analyses were performed using the Statistical Package for the Social Science (SPSS) version 17, software for Windows (SPSS, Chicago, IL, USA).

\section{Results}

One hundred seventy four patients were screened and 24 were randomized (Figure 1).

Two patients in each group did not complete the treatment protocol. In the $\mathrm{CIMT}_{1.5 \mathrm{~h} \_ \text {direct }}$ group, one patient moved far from the outpatient rehabilitation center and the other had financial limitations that impeded compliance with treatment three times a weak. One additional subject from this group had a fatal recurrent stroke before the last follow-up session at six months. In the $\mathrm{CIMT}_{3 \mathrm{~h} \text { direct }}$ group, one patient considered that the exercises were too difficult and the other one returned to work. Therefore, we analyzed data from nine patients in the $\mathrm{CIMT}_{1.5 \mathrm{~h} \_ \text {direct }}$ and ten patients in the $\mathrm{CIMT}_{3 \mathrm{~h} \_ \text {direct }}$ group.

Table 1 shows characteristics of the 19 subjects that completed the treatment and followup protocol. There were no significant differences in baseline characteristics between patients in the two groups.

In the $\mathrm{CIMT}_{1.5 \mathrm{~h} \text { direct }}$ group, patients wore the mitt for an average of $3.3 \pm 1.5$ daily hours and in the $\mathrm{CIMT}_{3 \mathrm{~h} \_ \text {direct }}$ group, for $2.8 \pm 1.2$ daily hours. However two patients in the

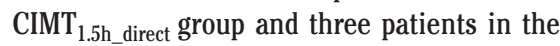
$\mathrm{CIMT}_{3 \mathrm{~h} \_d i r e c t}$ group did not log this information appropriately. 


\section{Primary outcome}

Figure 2A show results of EI for the MAL immediately after treatment and 6 months later. Immediately after treatment, there were no significant differences in EI results between the two groups $(\mathrm{P}=0.24)$. Likewise, no significant differences were observed between the groups, 6 months later $(\mathrm{P}=0.97)$, indicating comparable benefits from the two programs on real-life motor improvements.

\section{Secondary outcomes}

There were no significant differences in EI results regarding FMA scores between CIMT $_{1.5 h \_d i r e c t}$ group and the CIMT $_{3 \mathrm{~h} \text { _direct }}$ group immediately after treatment $(\mathrm{P}=0.60)$. Similarly, no significant differences between CIMT $_{1.5 h_{\text {_direct }}}$ and the CIMT $_{3 h_{\_} \text {direct group }}$ after 6 months $(\mathrm{P}=0.84)$ indicating similar effects of therapies on motor impairment (Figure 2B).

\section{Quality of life}

Figure 1C shows EI results. There were no significant differences between the two groups immediately after treatment $(\mathrm{P}=0.90)$ and at six months post-treatment $(\mathrm{P}=0.04)$.

\section{Discussion and Conclusions}

In the present study, improvements in motor

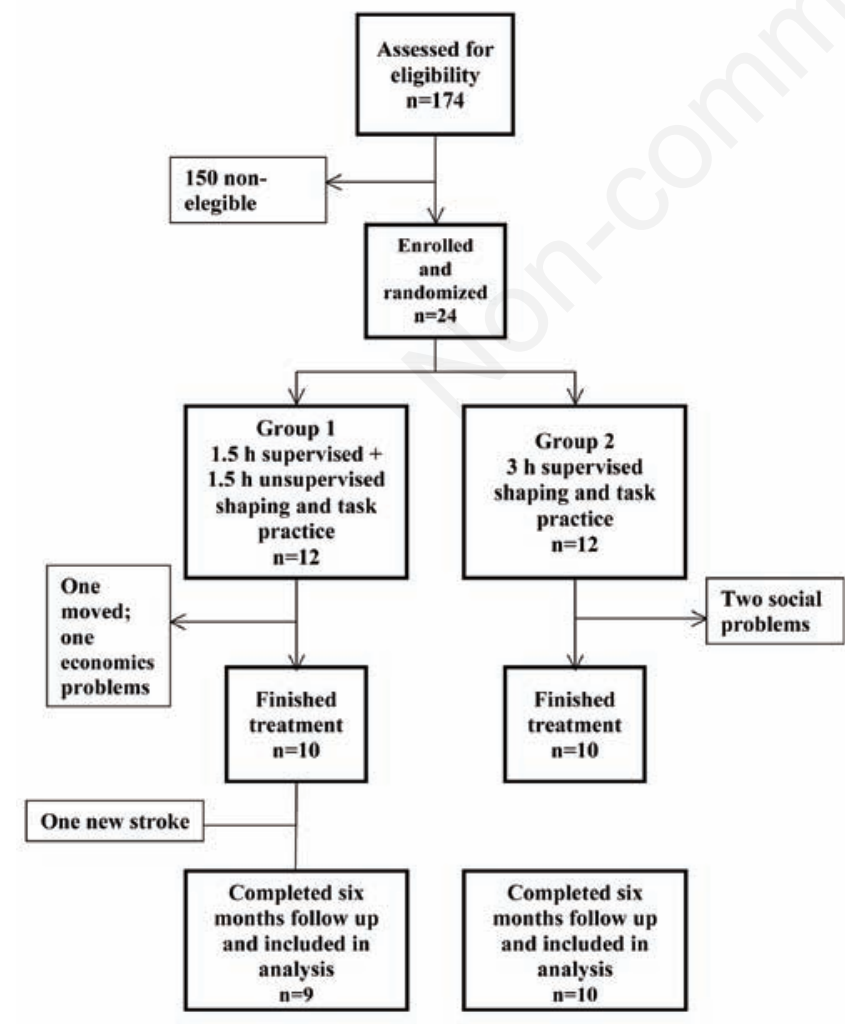

Figure 1. Flowchart of participants through the study. function, real-world arm use and quality of life did not differ significantly between treated groups receiving either 3 or 1.5 hours mCIMT supervised by a therapist. Results immediately after treatment and six months after that suggest that both protocols are equally efficient. Both groups had the same total training time (30 hours). However, CIMT $_{1.5 h \_d i r e c t}$ group may

Table 1. Characteristics of study participants.

\begin{tabular}{lccc} 
& CIMT 1.5h_direct & CIMT 3h_direct & P \\
Age, years, means (SD)* & $61.7(12.7)$ & $59.5(9.1)$ & 0.93 \\
Gender (men/women)** $^{*}$ & $6 / 3$ & $9 / 1$ & 0.55 \\
\hline Months after stroke, mean (SD)* & $27.6(20.9)$ & $35.3(33.8)$ & 0.71 \\
Type of stroke (ischemic/hemorrhagic)** & $7 / 2$ & $7 / 3$ & 0.55 \\
\hline Handedness (right/left)** $^{*}$ & $7 / 2$ & $9 / 1$ & 0.45 \\
Years of education* $^{*}$ & $5.0(1.7)$ & $6.6(3.2)$ & 0.44 \\
\hline Affected hemisphere (right/left)** & $4 / 5$ & $4 / 6$ & 0.60 \\
Motor Activity Log* $^{*}$ & $1.2(0.8)$ & $0.7(0.5)$ & 0.27 \\
\hline Stroke Specific Quality of Life Scale* & $163.1(39.3)$ & $143.3(25.3)$ & 0.21 \\
Fugl-Meyer assessment* & $53.4(10.8)$ & $51.4(8.6)$ & 0.44 \\
\hline
\end{tabular}

Data are mean and standard deviation (SD); *Mann-Whitney U test; **Fisher's Exact Test.
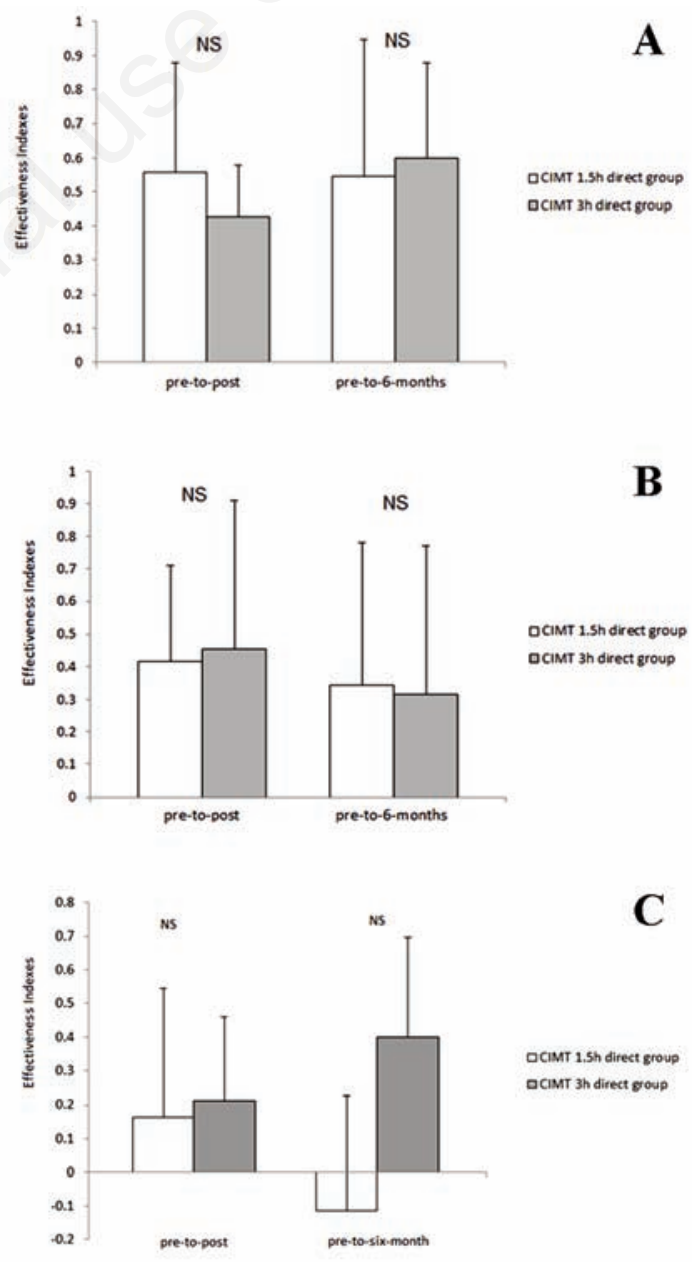

Figure 2. Effectiveness indexes results regarding Motor Activity Log (A), Fugl-Meyer Assessment (B) and Stroke Specific Quality of Life (C) scores between $\mathrm{CMIT}_{1.5 \mathrm{~h} \_ \text {direct }}$ group and $\mathrm{CIMT}_{3 \mathrm{~h} \_ \text {direct }}$ group pre-to-post treatment and pre-to- 6 months after treatment. Mann Whitney Test $(P<0.025)$. NS, not statistically significant. 
be considered less expensive due to less demand on therapist's time.

All previous studies that addressed the effectiveness of unsupervised practice at home also suggested similar effectiveness of different versions of modified CIMT. Supervision for only 25 to $50 \%$ of the time was as effective as standard one-to-one CIMT. ${ }^{36}$ Likewise, similar gains in motor skill and real-world arm use were reported after and one hour of supervised outpatient practice coupled with 5 hours of unsupervised practice during two weeks at home. ${ }^{37}$ Furthermore, An exclusive homebased mCIMT program supervised by an instructed family member was equally effective as a totally supervised protocol and persisted for the next six months. ${ }^{38}$

The development of flexible schedules may increase the number of treated patients. When questioned about their interest in participating in an original CIMT protocol, ${ }^{39} 68 \%$ of inquired patients said they were not interested because of concerns about the number of hours spent daily in therapy and the length of time required for wearing the restrictive device.

In mCIMT studies, participants are typically requested to wear the constraint from five to six hours daily, $14,16,40$ to $90 \%$ of the waking hours. ${ }^{41-43}$ Nevertheless, few studies reveal the real average number of daily hours with restraint in their results. ${ }^{40,44}$

In the present study, besides the declared mitt time use, some patients informed they consciously avoided the use of the less affected arm in ADLs during the treatment period, so the actual restraint time is somewhat difficult to measure precisely. The contribution of the restraint component of CIMT is in fact not fully established.45 A mCIMT study with random assignment comparing protocols with and without restraint in 24 patients found similar motor improvements after a one-year followup. ${ }^{46}$ A recent study, ${ }^{47}$ evolving 47 participants, showed no difference between two groups of post stroke patients treated with a combination of CIMT and physiotherapy plus different constraint regimens (sling versus voluntary constraint). It seems that using a sling were not an advantage over voluntary constraint.

As to functional outcomes, the present study indicates a substantial improvement in real world arm use after intervention in both groups. In a partially supervised CIMT program based on Taub's protocol, 37 gains in MAL were lost six months after therapy. Training in the present study was less intensive (three hours a day) and distributed over a longer period of time (21 days). This may have contributed to retention of therapeutic gains in both groups of patients. MAL scores at the six-month follow-up exhibited significant improvements in both groups with total score $>2.5$, indicating a perception of recovery above $50 \% .48,49$ The MAL results in our study were consistent with those observed in another protocol with the same intensity and duration. ${ }^{41}$

There is evidence that many post stroke patients do not use their paretic hand spontaneously due to learned non-use. ${ }^{50,51}$ The detected benefits in the FMA were partially but still meaningfully sustained after six months, although in CIMT $_{1.5 h \_d i r e c t}$ results did not reached statistical significance. FMA is a laboratory test that assesses motor function, showing what patients can do when requested.

Although CIMT protocols results in improved function it is not clear whether CIMT is necessary to reach the results reported in several studies or whether exposure to taskspecific and high-intensity training are the main components for recovery.52,53 Recently Taub et al.54 discussed the relevance of the transfer package that is a set of techniques to help transfer of therapeutic gains from laboratory to real life. The study concluded that the transfer package seems to be an important element of CIMT protocols increasing real-world treatment outcomes. The present study applied some principles of the transfer package like written assignment of practice at home, daily home diary and problem solving strategies to increase arm use in ADL. Health-related quality of life (HRQOL) is a multidimensional concept and can be impacted by several factors besides physical or mental status. ${ }^{55}$ A Chinese study with chronic stroke patients found that having a lower income was the strongest predicting variable for low HRQOL scores. 56 The effect of exercises on HRQOL in stroke survivors is not fully studied. A recent meta-analysis showed small to medium effects of exercises in HRQOL outcomes after intervention but not 12 to 24 weeks at follow-up. 57 In the present study, the absence of significant differences in EI for the SSQOL may be related to the small sample size, because within-group analysis showed significant improvements only in the $\mathrm{CIMT}_{3 \mathrm{~h} \text { group }}$.

The limitations of the present study are related to its small sample size and to the absence of a control group not receiving CIMT. Strict study criteria akin to those of EXCITE led to the inclusion of only $13.8 \%$ of screened subjects. Hence, our results should necessarily be considered preliminary. Future trials should involve a larger number of participants. This should allow investigating the ideal amount of daily training and the influence of each CIMT element in functional recovery. In conclusion, our modified CIMT protocols were feasible and well tolerated. Results in bothCIMT1.5h_direct group and $\mathrm{CIMT}_{3 \mathrm{~h} \_ \text {direct }}$ group were associated with similar improvements in real world arm use after stroke. As protocols involving less therapist supervision can reduce treatment costs, these results, if confirmed, could lead to a wider dissemination of CIMT as a standard treatment in large numbers of stroke survivors. This would be of special importance in developing countries, which should be prepared to take a greater part of the expected stroke burden in the future.

\section{References}

1. Feigin VL, Lawes CMM, Benett DA, Anderson CS. Stroke epidemiology: a review of population-based studies of incidence, prevalence, and case-fatality in the last 20th century. Lancet Neurol 2003;2:4353.

2. Gresham GE, Alexander D, Bishop DS, et al. American Heart Association prevention conference. IV. Prevention and rehabilitation of stroke. Rehabilitation. Stroke 1997;28:1522-6.

3. Sarti C, Rastenyte D, Cepaitis Z, Tuomiletho J. International trends in mortality from stroke, 1968-1994. Stroke 2000;31:1588-601.

4. Curioni C, Cunha CB, Veras RP, Andre C. The decline in mortality from circulatory diseases in Brazil. Rev Panam Salud Publica 2009;25:9-15.

5. Cabral NL, Gonçalves ARR, Longo AL, et al. Trends in stroke incidence, mortality and cases fatality rates in Joinville, Brazil: 1995-2006. J Neurol Neurosurg Psychiatry 2009;80:749-54.

6. Gauthier LV, Taub E, Mark VW, et al. Improvement after constraint induced movement therapy is independent of infarct location in chronic stroke patients: supplemental methods. Stroke 2009;40:2468-72.

7. Kwakkel G, Kollen BJ, van der Grond J, Prevo AJ. Probability of regaining dexterity in the flaccid upper limb. Impact of severity of paresis and time since acute stroke. Stroke 2003;34:2181-6.

8. Harvey RL. Improving post stroke recovery: neuroplasticity and task-oriented training. Curr Treat Opt Cardiovascul Med 2009;11:251-9.

9. Taub E, Miller NE, Novack TA. Technique to improve chronic motor deficit after stroke. Arch Phys Med Rehabil 1993;74:347-54.

10. Taub E, Crago JE, Uswatte G. Constraintinduced movement therapy: a new approach to treatment in physical medicine. Rehabil Psychol 1998;43:152-70.

11. Van der Lee JH, Wagenaar RC, Lankhorst GJ, et al. Forced use of the upper extremity in chronic stroke patients. Stroke 1999;30:2369-75.

12. Winstein CJ, Miller JP, Blanton S, et al. Methods for a multisite randomized trial to investigate the effect of constraint- 
induced movement therapy in improving upper extremity function among adults recovering from a cerebrovascular stroke. Neurorehabil Neural Repair 2003;17:13752.

13. Wolf SL, Winstein CJ, Miller JP, et al. Effect of constraint-induced movement therapy on upper extremity function 3 to 9 months after stroke: the EXCITE randomized clinical trial. JAMA 2006;296:2095-104.

14. Leung DPK, Ng AKY, Fong KNK. Effect of modified constraint-induced movement therapy for clients with chronic stroke in a community setting. Human Mov Sci 2009;28:798-808.

15. Page SJ, Sisto S, Levine P, McGrath RE. Efficacy of modified constraint - induced movement therapy in chronic stroke: a single- blinded randomized controlled trial. Arch Phys Med Rehabil 2004;85:14-8.

16. Page SJ, Levine P, Leonard AC. Modified constraint-induced therapy in acute stroke: a randomized controlled pilot study. Neurorehabil Neural Repair 2005;19:2732.

17. Boake C, Noser EA, Ro T, et al. Constraintinduced movement therapy during early stroke rehabilitation. Neurorehabil Neural Repair 2007;21:14-24.

18. Page SJ, Levine P, Leonard AC, et al. Modified constraint-induced movement therapy in chronic stroke: results of a single-blinded randomized controlled trial. Phys Ther 2008;88:333-40.

19. Wu CY, Lin KC, Chen CL, et al. A randomized controlled trial of modified constraintinduced movement therapy for elderly stroke survivors: changes in motor impairment, daily functioning, and quality of life. Arc Phys Med Rehabil 2007;88:273-8.

20. Folstein MF, Folstein SE, McHugh PR. Mini-mental state: a practical method for grading the cognitive state of patients for the clinician. J Psychiatric Res 1975;12:189-98.

21. Bohannon RW, Smith MB. Interrater reliability of a modified Ashworth scale of muscle spasticity. Phys Ther 1987;67:206-7.

22. Taub E, Uswatte G, King D, et al. A placebo controlled trial of constraint induced movement therapy for upper extremity after stroke. Stroke 2006;37:1039-45.

23. Taub E, Uswatte G, Pidikiti R. Constraintinduced movement therapy: a new family of techniques with broad application to physical rehabilitation: a clinical review. J Rehabil Res Dev 1999;36:237-51.

24. Uswatte G, Taub E, Morris D, et al. Reliability and validity of the upperextremity Motor Activity Log-14 for measuring real-world arm use. Stroke 2005;36:2493-6.

25. Hammer AM, Lindmark B. Responsiveness and validity of the motor activity $\log$ in patients during the subacute phase after stroke. Disabil Rehabil 2010;32:1184-93.

26. Sanford J, Moreland J, Swanson LR, et al. Reliability of the Fugl-Meyer assessment for testing motor performance in patients following stroke. Phys Ther 1993;73:44854.

27. Gladstone DJ, Danells CJ, Black SE. The Fugal-Meyer assessment of motor recovery after stroke: a critical review of its measurement properties. Neurorehabil Neural Repair 2002;16:232-40.

28. Maki T, Quagliato EMAB, Cacho EWA, et al. Estudo de confiabilidade da aplicação da escala de Fulg-Meyer no Brasil. Rev Bras Fisioter 2006;10;177-83.

29. Williams LS, Weinberger M, Harris L, Biller J. Measuring quality of life in a way that is meaningful to stroke patients. Neurology 1999;53:1839-43.

30. Williams LS, Weinberger M, Harris L, et al. Development of a stroke-specific quality of life scale. Stroke 1999;30:1362-9.

31. Lima RCM, Teixeira-Salmela LP, Magalhães LC, Gomes-Neto M. Propriedades psicométricas da versão brasileira da escala de qualidade de vida específica para acidente vascular encefálico: aplicação do modelo Rasch. Rev Bras Fisioter 2008;12:149-56.

32. Dahl TH. International classification of functioning, disability and health: an introduction and discussion of its potential impact on rehabilitation services and research. J Rehabil Med 2002;34:201-4.

33. Lin KC, Chung HY, Wu CY, et al. Constraint-induced movement therapy versus control intervention in patients with stroke. Am J Phys Med Rehabil 2010;89:177-85.

34. Shah S, Vanclay F, Cooper B. Efficiency, effectiveness and duration of stroke rehabilitation. Stroke 1990;21:241-6.

35. Klugler C, Altenhöner T, Lochner P, Ferbert A. Does age influence early recovery from ischemic stroke? J Neurol 2003;250:67681.

36. Taub E, Lum PS, Hardin P, et al. AutoCITE: automated delivery of CI therapy with reduced effort by therapists. Stroke 2005;36:1301-4.

37. Richard L, Rothi LJG, Davis S, et al. Limited dose response to constraintinduced movement therapy in patients with chronic stroke. Clin Rehabil 2006;20:1066-74.

38. Barzel A, Liepert J, Haevernick K, et al. Comparison of two types of constraintinduced movement therapy in chronic stroke patients: a pilot study. Res Neurol Neurosci 2009;27:673-80.

39. Page SJ, Levine P, Sisto S. Stroke patient's and therapist's opinions of constraintinduced movement therapy. Clin Rehabil
2002;16:55-60.

40. Lin KC, Wu CY, Wei TH, et al. Effects of modified constraint-induced movement therapy on reach-to-grasp movements and functional performance after chronic stroke: a randomized controlled trial. Clin Rehabil 2007;21:1075-86.

41. Sterr A, Elbert T, Berthold I, et al. Longer versus shorter daily constraint-induced movement therapy of chronic hemiparesis: an exploratory study. Arch Phys Med Rehabil 2002;83:1374-7.

42. Wittenberg GF, Chen R, Ishii $\mathrm{K}$, et al. Constraint-induced therapy in stroke: magnetic-stimulation motor maps and cerebral activation. Neurorehabil Neural Repair 2003;17:48-57.

43. Wolf SL, Thompson PA, Winstein CJ, et al. The EXCITE stroke trial, comparing early and delayed constraint-induced movement therapy. Stroke 2010;41:2309-15.

44. Porter K. Constraint-induced movement therapy for people following stroke in an outpatient setting. NZ J Phys 2004;32:1119.

45. Sterr A, Saunders A. CI therapy distribution: theory, evidence and practice. Neurorehabil 2006;21:97-105.

46. Brogärdh C, Vestiling M, Sjölund BH. A 1 year follow-up after shortened constraintinduced movement therapy with and without mitt poststroke. Arch Phys Med Rehabil 2010;91:460-4.

47. Krawczyk M, Sidaway M, Radwanska A, et al. Effects of sling and voluntary constraint during CIMT for the arm after stroke: a randomized, prospective, single-centre, blinded observer rated study. Clin Rehabil 2012;26:990-8.

48. Fritz SL, George SZ, Wolf SL, Ligth KE. Participant perception of recovery as criterion to establish importance of improvement for CIMT outcome measures: a preliminary study. Phys Ther 2007;87:170-8.

49. Park SW, Wolf SL, Blanton S, et al. The EXCITE trial: predicting a clinically meaningful motor activity log outcome. Neurorehabil Neural Repair 2008;22:48693.

50. Sterr A, Freivogel S, Schmalohr D. Neurobehavioral aspects of recovery: assessment of the learned nonuse phenomenon in hemiparetic adolescents. Arch Phys Med Rehabil 2002;83:1726-31.

51. Uswatte G, Taub E. Implications of the learned non use formulation for measuring reabilitations outcomes: lessons from CIMT. Rehabil Psychol 2002;50:34-42.

52 . Viana R, Teasell R, Barriers to the implementation of constraint-induced movement therapy into practice. Tops Stroke Rehabil 2012;19:104-14.

53. Sterr A, Saunders A. CI therapy distribution: theory, evidence and practice. 
Neurorehabil 2006;21:97-105.

54. Taub E, Uswatte G, Mark VW, et al. Method for enhancing real-world use or a more affected arm in chronic stroke. Transfer package of constraint-induced movement therapy. Stroke 2013;44:1383-8.
55. Carod-Artal FJ, Egidio JA. Quality of life after stroke: the importance of a good recovery. Cerebrovasc Dis 2009;27:204-14.

56. Delcourt C, Hackett $\mathrm{M}, \mathrm{Wu} \mathrm{Y}$, et al. Determinants of quality of life after stroke in China- the ChinaQuest (quality evalua- tion of stroke care and treatment) study. Stroke 2011;42:433-8.

57. Chen MD, Rimmer JH. Effects of exercises on quality of life in stroke survivors: a meta-analysis. Stroke 2011;42:827-32. 